\title{
Reliability of CoaguChek Pro II Point of Care System in Outpatient Setting in Farwaniya Hospital, Kuwait
}

\author{
Aida Thabet ${ }^{1,2}$, Danah Al-Shatti ${ }^{2}$, Mohamed Elrahwan ${ }^{2, ~ * ~}$ \\ ${ }^{1}$ Department of Clinical Pathology, Banha University, Banha, Egypt \\ ${ }^{2}$ Department of Hematopathology, Farwaniya Hospital, Farwaniya, Kuwait
}

Email address:

mrahwan69@gmail.com (M. Elrahwan)

${ }^{*}$ Corresponding author

\section{To cite this article:}

Aida Thabet, Danah Al-Shatti, Mohamed Elrahwan. Reliability of CoaguChek Pro II Point of Care System in Outpatient Setting in Farwaniya Hospital, Kuwait. American Journal of Laboratory Medicine. Vol. 6, No. 3, 2021, pp. 31-36. doi: 10.11648/j.ajlm.20210603.11

Received: April 16, 2021; Accepted: May 5, 2021; Published: May 14, 2021

\begin{abstract}
Point of care testing (POCT) coagulometers are widely used for international normalized ratio (INR) monitoring for patients on vitamin $\mathrm{K}$ antagonists (VKA) therapy. In our study, we investigated the accuracy and reliability of CoaguChek pro II (Roche Diagnostics) as an alternative for standard laboratory testing (SLT) by ACL TOP 500 top system in outpatient department setting. Methods: We enrolled a total of 174 INR results in our study which were measured by CoaguChek ProII and ACL TOP 500 top. The three arms of the study were: INR $<3.5,3.5-4.4$, and $\geq 4.5$. The results were compared using Passing Bablok regression analysis and Bland-Altman plot. The agreement between the two methods was further evaluated to demonstrate the impact on dosing decision. Furthermore, the degree of patient satisfaction with POCT in our INR clinic was assessed by participating in a survey. Results: The overall correlation of INR measurements between POCT and SLT in our study was strong $(r=0.95)$, however, the correlation between the two methods in the 3.5-4.4 arm was moderate $(\mathrm{r}=0.502)$. The overall agreement between the two methods in all three arms of the study in terms of dosing decision was good (Kappa $=0.862$ ), with only $12.6 \%$ of INR measurements showing a difference in dosing decision. Ninety-Seven percent of all INR values measured by CoaguChek Pro II within therapeutic range (INR<3.5) were within 0.5 INR units when compared to ACL TOP 500 Top. Furthermore, we concluded that more than 90\% of the patients in our center were satisfied with the POC service. Conclusion: We concluded that POCT is a good alternative to SLT in INR values falling in therapeutic and supra-therapeutic ranges; however, additional comparative studies investigating the accuracy and reliability between the two methods for INR results between 3.5-4.4 will add to the current body of knowledge. Furthermore, the majority of patients were satisfied with the service provided in the POC INR clinic.
\end{abstract}

Keywords: Point-of-care, CoaguChek Pro II, INR Monitoring, Patient Satisfaction

\section{Introduction}

Point-of-care (POC) INR monitoring devices (otherwise known as 'coagulometers') are small and portable instruments which measure the time to clot formation after fingerstick blood sampling for patients on vitamin $\mathrm{K}$ antagonists (Coumadin, warfarin). Not only can POC devices be used in hospital setting, but they can also be used by patients for self-monitoring/management [1]. For many years, prothrombin time was the main laboratory investigation to monitor warfarin therapy; however, it has been replaced by a more standardized method, the INR, due to the variability of thromboplastin reagents used [2]. POC testing (POCT) provides an alternative to standard laboratory testing (SLT) and venepuncture, by obtaining a fingerstick sample of whole blood to achieve an INR result instantly, and thus allowing for a rapid dose adjustments of warfarin with less volume of blood drawn and shorter time for hospital visits when compared to the standard venepuncture method, which might have a positive impact on patient's preference of POCT [3]. Warfarin monitoring by POCT can be performed by one of two ways: either by patient self-testing at home, or as in our study, in outpatient setting [1]. There are multiple POC devices available currently in the market [4]. The coagulometer investigated in our study is CoaguChek Pro II, which was launched by Roche in 2015. As a POC device, CoaguChek Pro II has the advantage of a built-in WIFI connectivity, making 
the transfer of patients' data easier from the device to the patient's electronic health records, such as the Labortory Information System (LIS) [5].

The accuracy of CoaguChek system has been investigated in several countries, with reliability and accuracy of which being questionable above INR of 4.5 or even 3.5 [2, 6, 7]. Although laboratory testing is less expensive than POC for INR measurement, the latter has been found to be cost-effective [4, 8]. Patient satisfaction has been assessed in several studies with the better treatment satisfaction being in favour of POC compared to SLT, which ultimately has a positive impact both on thromboembolic events and anticoagulation control [3].

The aim of the present study was to: (1) validate the INR results obtained with CoaguChek Pro II at three levels of INR ranges $(0-3.5,3.5-4.4$ and $\geq 4.5)$ (2) to evaluate the 0.5 INR concordance between POCT and SLT (3) and to determine the degree of patient's satisfaction with the INR clinic since implementing the POCT as the standard of care rather than SLT.

\section{Methods and Material}

This study was performed in the anticoagulation clinic in one of the main six governmental hospitals in Kuwait, Farwaniya hospital, which is currently run by a hemato-pathology team. A total of 174 patients were recruited in this retrospective observational study. All patients visiting the INR clinic were on longterm warfarin and came for INR monitoring and dose adjustment. The cases enrolled in the study were the first five patients on the INR clinic OPD list, based on the number given for the patient by clerk. The cases were recruited daily from Sunday to Thursday from the date the POCT has been implemented. All had their INR checked by the test method CoaguChek Pro II and counterchecked by SLT using ACL TOP 500. There were three arms for the study based on the INR result on CoaguChek: (1) INR <3.5 ( $\mathrm{n}=96$ ), (2) INR between 3.5-4.4 $(\mathrm{n}=22)$, and INR $\geq 4.5(\mathrm{n}=56)$. All samples collected in the INR clinic were by a single trained phlebotomist with 5 years experience for blood collection and clinical laboratory tests. The patient had a finger stick sample collected for CoaguChek. Within fifteen minutes, the patient had phlebotomy using a vaccutainer, with $2.7 \mathrm{ml}$ of patient's blood collected in a sodium citrate tube with $3.2 \%$ citrate concentration. The sample was centrifuged at 4000 RPM for 5 minutes using Allegra centrifuge. After centrifugation was completed, the sample was placed in a sample rack before inserting it inside the ACL TOP 500 machine. As for the satisfaction with the INR service since implementing the POC service, blank questionnaire sheets were available outside the clinic, and 96 anonymous and voluntary questionnaires were collected within the same duration of the study, with the date being the only data written by the patient beside the answers to the questions.

\section{Statistical Analysis}

Mean (SD) for continuous data and frequency (percent) for categorical data were reported as descriptive statistics. The Passing-Bablok regression models along with the Pearson's correlation coefficients were conducted to determine the association between INR values which measured by CoaguCkek Pro II and ACL TOP 500. The Bland-Altman and Kappa agreement methods were used to test the similarity between measurements of INRs which evaluated by instruments.

The above analyses were conducted for all measurements $(\mathrm{n}=174)$, measurements with INR $<3.5(\mathrm{n}=96)$, INR in 3.5-4.4 $(\mathrm{n}=22)$, and INR $\geq 4.5(\mathrm{n}=56)$.

STATA version 13 was utilized for statistical analysis and $P$ values $<0.05$ were considered statistically significant.

\section{Results}

In the total of 174 measurements, the mean (SD) of INR values based on the two measurement methods of CoaguCkek Pro II and ACL TOP 500 was 3.78 (2.16), (ranged from 1.0 to 8.0) and 3.51 (1.72) (ranged from 1.1 to 8.8), respectively.

The frequency distribution of all INR values measured by the two methods was categorized in 4 groups $(<2,2-4,4-6$, and $>6$ ) (Figure 1).

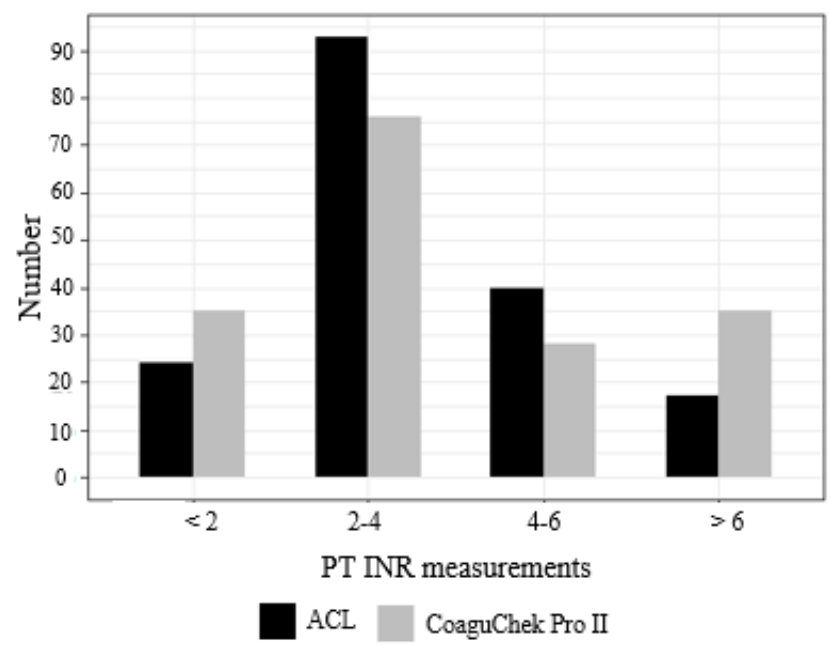

Figure 1. The distribution of prothrombin time (PT) international normalized ratio (INR) values which measured by CoaguChek Pro II and ACL TOP 500.

Passing-Bablok regression analysis as depicted in Figure 2 (a-d) shows the relationship between CoaguChek Pro II and ACL TOP 500 measurements for all data and subgroups, indicates good overall associations without significant deviations from linearity.

In the total of 174 measurements of INR, the Pearson's correlation coefficient between CoaguChek Pro II and ACL TOP 500 was strong $\mathrm{r}=0.95$ (95\% CI, 0.933-0.962).

Furthermore, the Pearson's correlation coefficients in the ranges of INR $<3.5$ and INR $\geq 4.5$, were strong, $r=0.889$ $(95 \%$ CI, $0.838-0.925)$, and $r=0.784 \quad(95 \%$ CI, 0.657-0.868), respectively. The correlation between CoaguChek Pro II and ACL TOP 500 was moderate, $r=$ 0.502 (95\% CI, 0.102-0.762) in the range of INR 3.5-4.4. 
The Bland-Altman analysis results to assess agreement between two measurements of INR (CoaguChek Pro II and ACL TOP 500) was displayed in Figure 3. The mean difference between the INR measurements by ACL TOP 500 and CoaguChek Pro II was 0.28 with the $95 \%$ agreement limits of -1.19 to 1.75 and $12 / 174(6.90 \%)$ of data was outside the limits of agreement. The agreement analysis in INR subgroups revealed that the mean difference between INR values measured by the two methods in the range of INR $<3.5$ was -0.11 (agreement limits: -0.61 to 0.40 ), and $4 / 96(4.16 \%)$ of data was outside the limits of agreement. In the range of INR 3.5-4.4, the mean difference was 0.03 (agreement limits: -0.77 to 0.83 ), and $2 / 22(9.09 \%$ ) of data was outside the limits of agreement. In the range of INR $\geq 4.5$ this difference was -0.59 with the agreement limits of -0.89 to 2.68 and $3 / 56(5.35 \%)$ of data was outside the limits of agreement. (Figure 3).

The agreement of INR measurements between CoaguChek Pro II and ACL TOP 500 was further evaluated in relation to dosing decision in all three arms of the study assessed $(<3.5$, $3.5-4.4$, and $\geq 4.5)$. The overall agreement was good (kappa $=0.862$; 95\%CI: $0.808-0.915$ ) and 22/174 (12.6\%) of all INR values displayed a difference in dosing decision between the two measurement methods.

In terms of INR difference between the two methods, the percentage of cases whose INR difference was $<0.5$ INR units in the INR group of $<3.5,3.5-4.4$, and $\geq 4.5$, was $97 \%$, $82 \%$, and $21 \%$, respectively (Figure 4 ).

As for patient satisfaction; $93 \%$ of all patients who participated in the survey were very satisfied with the POC system for INR monitoring (Figure 5).

\section{Discussion}

While multiple comparative studies on POC coagulometers and SLT exist in the literature, they all differ in the study designs, manufacturer of each method, and statistical analyses, giving rise to various conclusions and interpretation of the used tools in the study [9-13]. In this observational retrospective study, we investigated the analytical and clinical performance of CoaguChek POC device as an instant and convenient alternative method to SLT for warfarin therapy monitoring with a wide range of INR results, ranging from INR of 1 to 8. Transferring INR results from CoaguChek Pro II to the LIS, facilitated the collection of data; this feature reduced human error to a minimum [5].

Although CoaguChek test strips are calibrated to safely report INR values up to 4.5 , variability of INR results between POCT and SLT has been found in multiple studies above an even lower threshold of 3.5 [2, 4, 6, 7]. On the contrary, Lawrie and colleagues concluded that INR values on CoaguChek system between 4.6 and 7.9 were comparable to laboratory methods [9]. In this study, we used Passing-Bablok regression analysis to determine overall associations between the two methods for all arms of the study, which showed good association without significant diversion from linearity.

Furthermore, there was a good agreement between
CoaguChek Pro II and ACL TOP 500, with 95\%, 50\%, and 91\% agreement in the $<3.5,3.5-4.5$, and $\geq 4.5$ arm, respectively, and only $22 / 174(12.6 \%)$ cases showed discrepancy between the two methods. This was further emphasized by Pearson's correlation coefficients being strong in the ranges of INR $<3.5$ and $\geq 4.5$, whereas that of the INR range of 3.5-4.4 was moderate.

We used Bland-Altman difference plot in our study to assess agreement between CoaguChek Pro II and ACL TOP 500. It emphasizes that the mean difference in INR values between the two methods was 0.28 within the $95 \%$ agreement limits, and only12/174 (6.9\%) of data was outside the agreement limits. The mean difference between the INR methods in the three arms of the study: $<3.5,3.5-4.5$, and $\geq 4.5$, was $-0.11,0.03$, and -0.59 , respectively.

On clinical grounds, a difference of 0.5 INR units or less between POCT and SLT has been found to be an acceptable variation [7, 9, 10]. In an Australian study, $88 \%$ of CoaguChek cases had concordance less than 0.5 INR units when compared to the laboratory method [12]. Seventy-three percent of all the cases enrolled in our study were within 0.5 INR units on both CoaguChek Pro II and SLT. For those within the therapeutic range (i.e. INR $<3.5 \mathrm{arm}$ ), $97 \%$ of cases had INR difference of $<0.5$ INR units. This was comparable to a study conducted in South Africa in which $94 \%$ of the therapeutic INR values had a difference of INR values $<0.5$ units [7]. As for the other two arms of the study, we concluded that the percentage of cases with difference in INR values being $<0.5$ was $82 \%$ and $21 \%$ for the $3.5-4.5$ arm and $\geq 4.5$ arm, respectively.

Quality of life (QoL) measures were investigated in several studies, the majority of which favor POC INR monitoring in terms of patient satisfaction and QoL, compared to other methods [3]. Derek et. Al investigated the acceptance of POCT as an alternative option for INR measurement, and concluded that patients favoured capillary blood sampling by finger prick as opposed to veni-puncture for INR monitoring [14]. Although the cost of POCT is roughly three times that of SLT in our center, we established that $93 \%$ of the patients in our INR clinic are satisfied with POCT as a method for INR monitoring. This was comparable to a study conducted in South Carolina which demonstrated 95\% preference of POCT when compared to venepuncture for INR monitoring [15].

\section{Conclusion}

In summary, we compared INR results generated on CoaguChek Pro II with ACL TOP 500 to establish the degree of reliability of the former in the anticoagulation clinic setting. The three arms of the study were INR $<3.5,3.5-4.4$, and $\geq 4.5$. We established good association between the two methods in the $<3.5$ and $\geq 4.5$ arms. As for the 3.5-4.4 arm, the agreement was moderate, which could be due to the small sample size. Furthermore, almost all the values falling within the therapeutic range, had INR difference $<0.5$ INR units. As regards to patient satisfaction, we concluded that the majority of patients were satisfied with POCT for INR monitoring. 


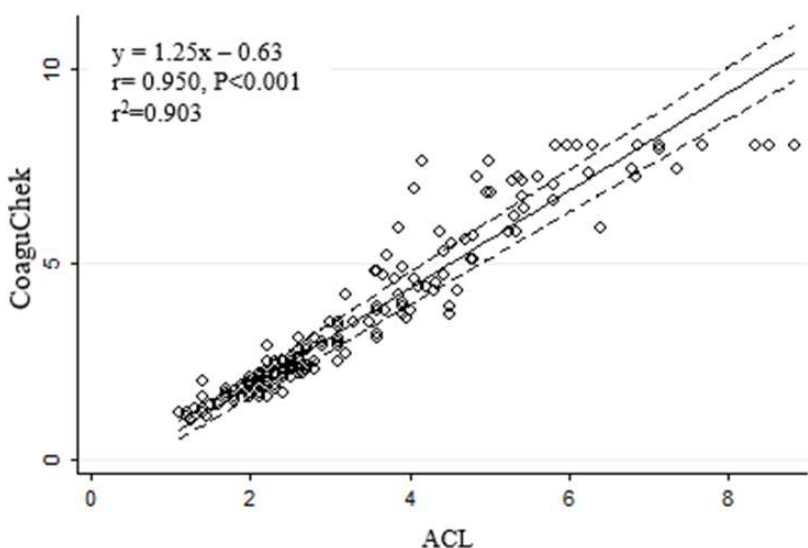

a. All data

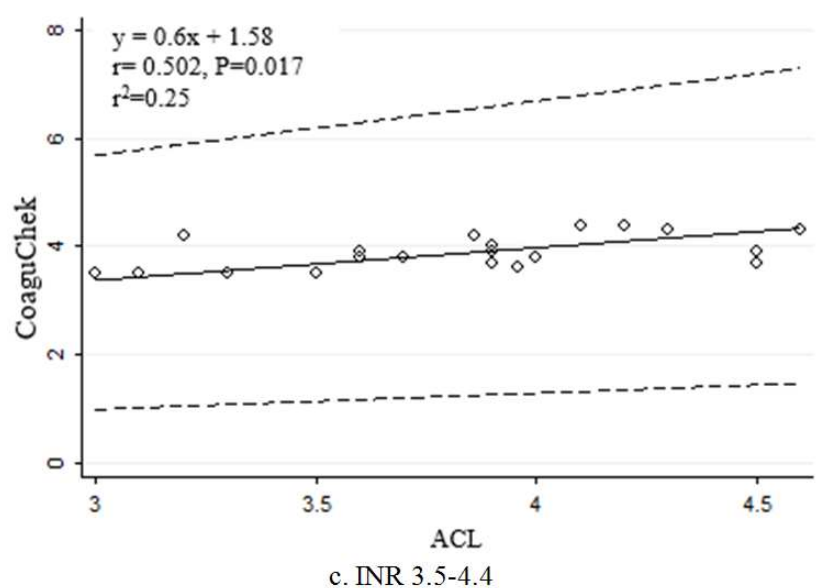

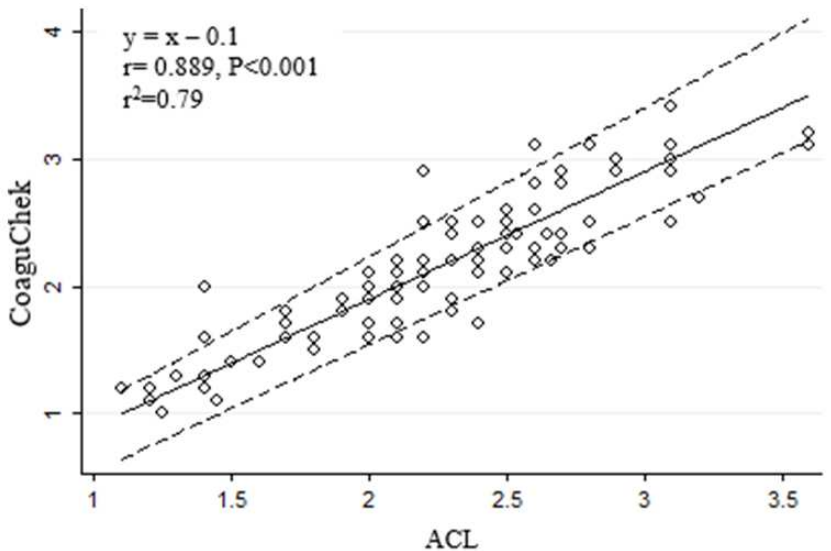

b. INR $<3.5$

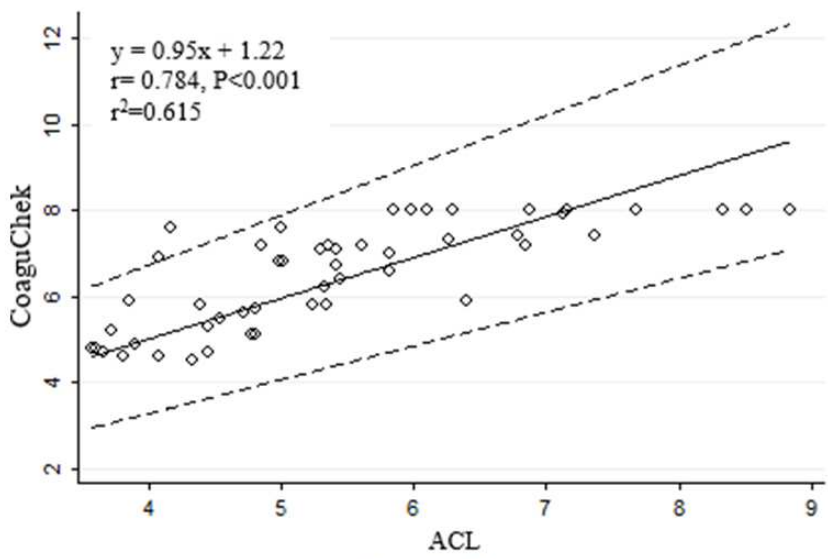

d. $\operatorname{INR} \geq 4.5$

Figure 2. Passing/Bablok regression analysis results: Comparison of INR metrics measured by CoaguChek Pro II and the ACL TOP 500. The solid lines indicate the regression lines, and the dashed lines indicate the $95 \%$ confidence interval (CI).

(a) Total data ( $\mathrm{n}=174)$ : The slope of regression line is $1.25(95 \% \mathrm{CI}=1.18$ to 1.31$)$ and an intercept of -0.63 ( $95 \% \mathrm{CI}=-0.78$ to -0.46$)$, Lin's concordance correlation coefficient of absolute agreement is 0.92 .

(b) INR $<3.5(\mathrm{n}=96)$ : The slope of regression line is $1.0(95 \% \mathrm{CI}=1.0$ to 1.17$)$ and an intercept of $-0.10(95 \% \mathrm{CI}=-0.40$ to -0.10$)$, Lin's coefficient of agreement is 0.87 .

(c) INR $3.5-4.4(\mathrm{n}=22)$ : The slope of regression line is $0.6(95 \% \mathrm{CI}=1.0$ to 1.17$)$ and an intercept of $1.58(95 \% \mathrm{CI}=0.1$ to 2.69$)$, Lin's coefficient of agreement is 0.45 .

(d) INR $>=4.5(\mathrm{n}=56)$ : The slope of regression line is $0.95(95 \% \mathrm{CI}=0.78$ to 1.16$)$ and an intercept of $1.22(95 \% \mathrm{CI}=0.15$ to 2.11$)$, Lin's coefficient of agreement is 0.58 .

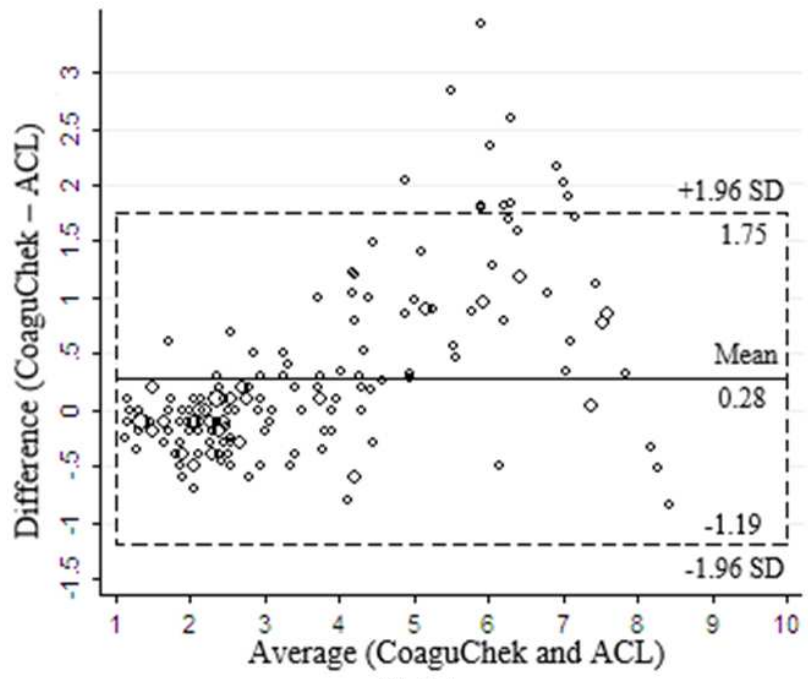

a. All data

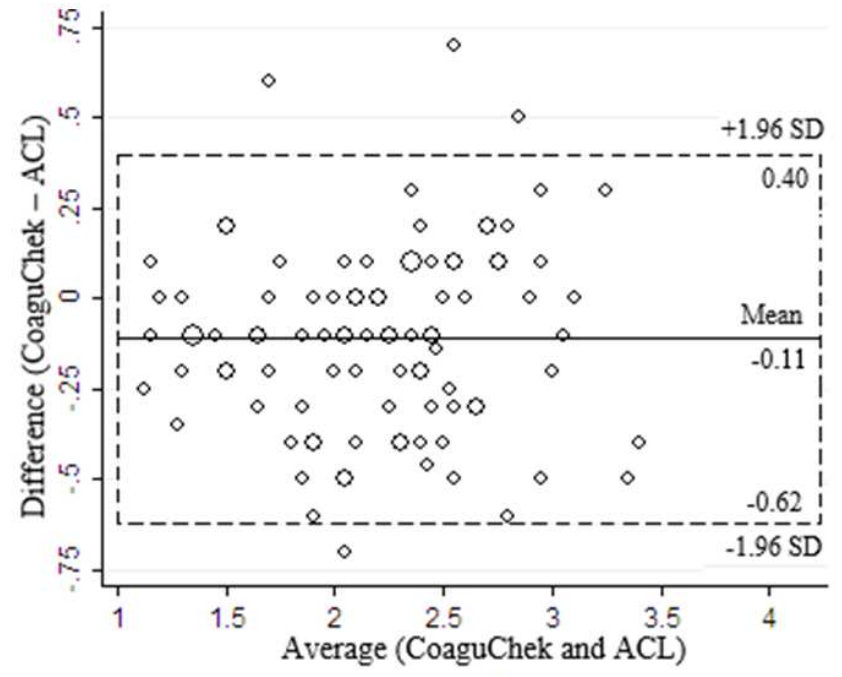

b. INR $<3.5$ 

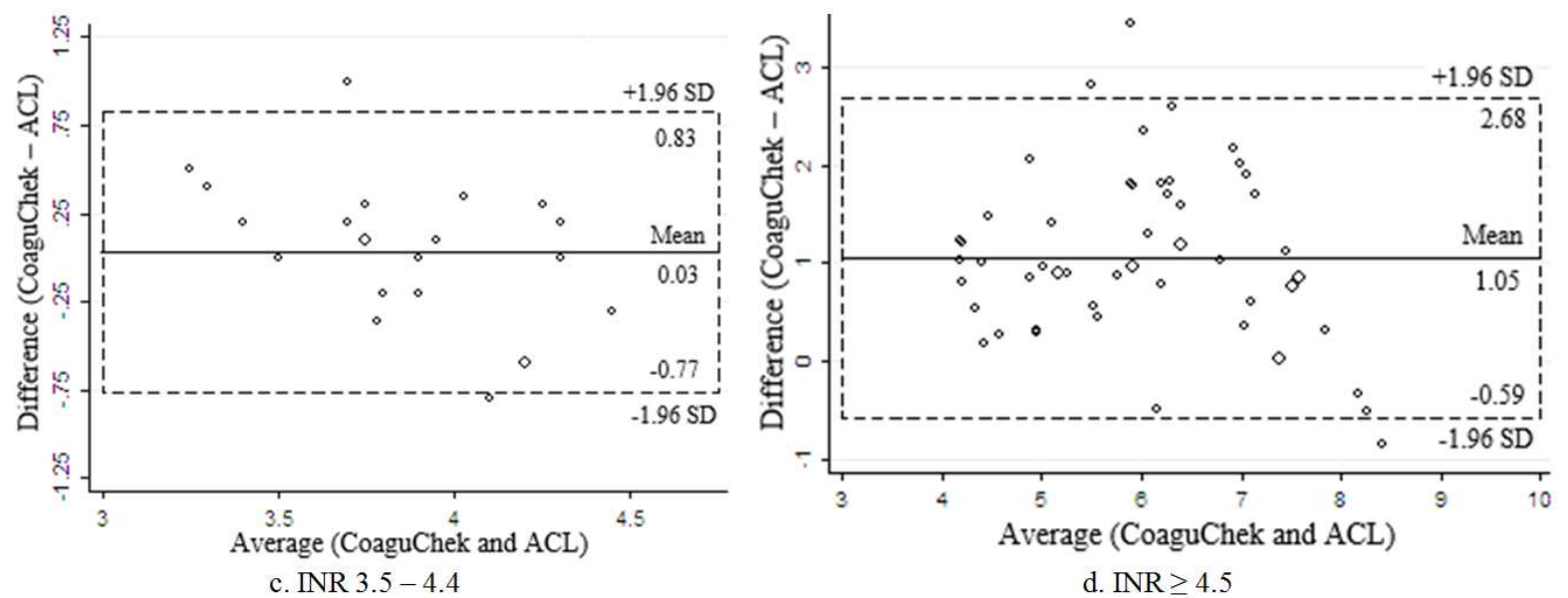

Figure 3. Comparison of INR measurements between the CoaguChek Pro II and ACL TOP 500 using Bland-Altman plots in the range of (a) total data, (b) INR $<3.5$, (c) INR 3.5-4.4, and (d) INR $\geq 4.5$.

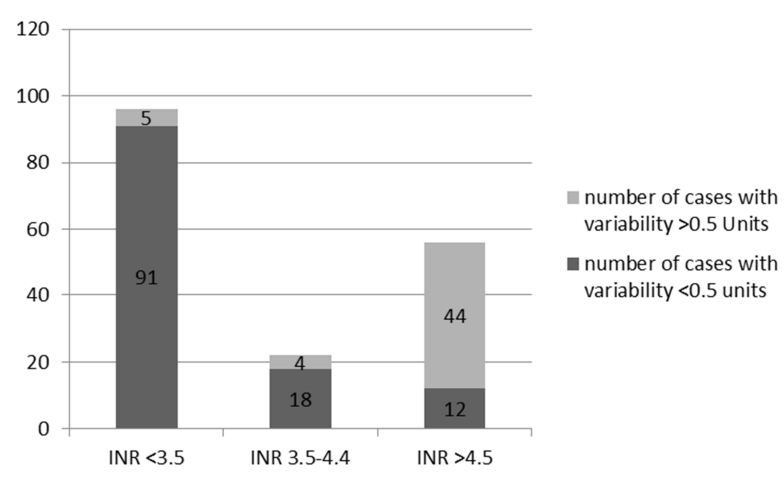

Figure 4. Variability (in INR units) between Coaguchek and ACL TOP 500 with the number of cases illustrated for each group.

$y$-axis represents the difference between two values and plotted against the average of ACL TOP 500 and CoaguChek Pro-II ( $x$-axis). The solid lines represent the mean differences of INR measurements for two methods, and the dashed lines represent mean difference \pm 1.96 standard deviation (SD).

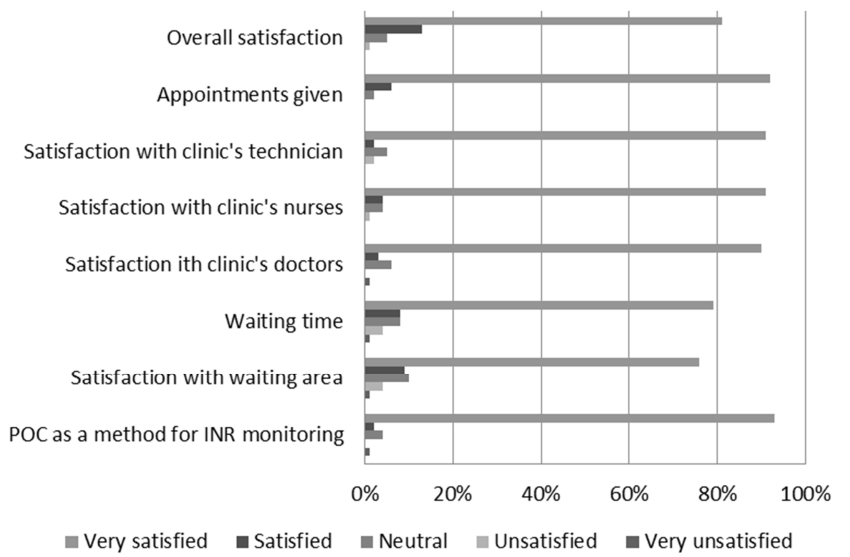

Figure 5. Patient satisfaction survey with POC/INR clinic.

Table 1. Agreement of INR measurements between CoaguChek Pro II and ACL TOP 500.

\begin{tabular}{lllll}
\hline \multirow{2}{*}{ ACL TOP 500 } & CoaguChek Pro II & & & Total (\%) \\
\cline { 2 - 5 } & INR $<$ 3.5 & INR 3.5 - 4.4 & INR $\geq$ 4.5 & $98(56.3)$ \\
\hline INR $<3.5$ & 94 & 4 & 12 & $28(16.1)$ \\
INR 3.5 -4.4 & 2 & 14 & 44 & $48(27.6)$ \\
INR $\geq 4.5$ & 0 & 4 & $56(32.2)$ & 174 \\
Total (\%) & $96(55.2)$ & $22(12.6)$ & & \\
\hline
\end{tabular}

Kappa value was 0.862 (95\% CI: 0.808 to 0.915$)$.

The agreement of INR measurements between CoaguChek Pro II and ACL TOP 500 was evaluated in relation to dosing decision in all three arms of the study assessed $(<3.5,3.5-4.4$, and $\geq 4.5$ ). The overall agreement was good (kappa $=0.862$; 95\%CI: $0.808-0.915)$ and 22/174 (12.6\%) of all INR values displayed a difference in dosing decision between the two measurement methods.

\section{References}

[1] Warfarin: Point-of-Care INR Monitoring, Thrombosis Canada 2017.
[2] Sade Adedeji-Zakari, The CoaguChek S System, U.S. Pharmacist, Feb 20, 2007.

[3] Point Of Care International Normalized Ratio (INR) Monitoring Devices for Patients on Long-term Oral Anticoagulation Therapy, Ontario Health Technology Assessment Series, 2009; Vol. 9, No. 12.

[4] Guidance on the Use of Point -of-Care Testing of International Normalized Ratio for Patients on Oral Anticoagulant Therapy, CADTH Optimal Use Report, July 2014, Vol. 3, Issue 1C.

[5] CoaguChek Pro II, Roche Diagnostics, November 25, 2015

[6] Deviations of high $(>4.5)$ CoaguChek INR values due to calibration with WHO reference standard $\mathrm{rTF} / 16$, Roche Diagnostics, September 20, 2018. 
[7] Benade E. L., Jacobson B. F., Louw S., Schapkaitz E. Validation of the CoaguChek XS international normalised ratio point-of-care analyser in patients at Charlotte Maxeke Johannesburg Academic Hospital, South Africa. South African Medical Journal 2016; 106 (3): 280.

[8] Point-of-Care INR Testing Compared with Lab INR Testing: What Does The Evidence Say?, CADTH 2015.

[9] Lawrie AS1, Hills J, Longair I, Green L, Gardiner C, Machin SJ, Cohen H. The clinical significance of differences between point-of-care and laboratory INR methods in over-anticoagulated patients. Thromb Res. 2012 Jul; 130 (1): $110-114$.

[10] Sunderji R1, Gin K, Shalansky K, Carter C, Chambers K, Davies C, Schwartz L, Fung A. Clinical impact of point-of-care vs laboratory measurement of anticoagulation. Am J Clin Pathol. 2005 Feb; 123 (2): 184-188.

[11] Fitzmaurice DA, Machin SJ. Recommendations for patients undertaking self management of oral anticoagulation. BMJ. 2001 Oct 27; 323 (7319): 985-989.
[12] Ignjatovic V1, Barnes C, Newall F, Hamilton S, Burgess J, Monagle P. Point of care monitoring of oral anticoagulant therapy in children: comparison of CoaguChek Plus and thrombotest methods of venous international normalized ratio. Thromb Haemost. 2004 Oct; 92 (4): 734-737.

[13] Jackson SL, Bereznicki LR, Peterson GM, Marsden KA, Jupe DM, Vial JH, Rasiah RL, Misan G, Williams SM. Accuracy and clinical usefulness of the near-patient testing CoaguChek S international normalized ratio monitor in rural medical practice. Aust J Rural Health. 2004 Aug; 12 (4): 137-142.

[14] Chapman DC1, Stephens MA, Hamann GL, Bailey LE, Dorko CS. Accuracy, clinical correlation, and patient acceptance of two handheld prothrombin time monitoring devices in the ambulatory setting. Ann Pharmacother. 1999 Jul-Aug; 33 (7-8): 775-780.

[15] Amy N. Thompson, Kelly R. Ragucci, Joli D. Fermo, and Heather P. Whitley. Evaluation of patient perceptions and outcomes related to anticoagulation point-of-care testing in ambulatory care clinics. Pharm Pract (Granada). 2009 Oct-Dec; 7 (4): 213-217. 